\title{
Improved techniques of measuring accurate electron - molecule cross sections near threshold and over a large angular range
}

\author{
Michael Allan \\ Department of Chemistry, University of Fribourg, CH-1700 Fribourg, Switzerland
}

\begin{abstract}
Methods to measure absolute differential elastic and vibrational excitation cross sections using an electron spectrometer with a magnetic angle changer are discussed. Emphasized are the need to by-pass drifts and to properly correct for the instrumental response function when angle of detection or electron energy are varied. The results are illustrated with cross sections in nitrogen, methane and neopentane. The good agreement of the results with the experimental data from other leading groups (taken mostly without the angle changer) and with some theories indicate that the measurements are reliable. The angle-integrated data agrees well with the results of electrontransport experiments. Good agreement with theory is obtained for nitrogen, even in the difficult low-energy region. Electronic excitation in rare gases is also briefly mentioned.
\end{abstract}

Keywords: electron-molecule collisions, cross sections, electron scattering, plasma modeling PACS: $34.80 . \mathrm{Gs}$

\section{INTRODUCTION}

Plasmas are important in nature and in many areas of technology and it is therefore desirable to be able to model them. Despite the long effort invested into the quest, this goal has not yet been fully achieved. The reason is, in part, the lack of adequate sets of cross sections. Any detailed model must include information on the elementary plasma processes, the electron - atom and electron - molecule collisions, in the form of quantitative (absolute) cross sections. Complete sets of cross sections are, despite the long history of electron - molecule collision measurements, very rarely available. The available cross sections often miss certain processes, do not cover certain ranges of parameters, in particular low energies, near threshold regions, and forward and backward angles.

The perhaps most important event in the effort to improve this situation has been the invention of the magnetic angle changer (MAC) [1,2]. Despite the availability of the MAC, however, measuring differential cross sections at very low and very large angles remains far from trivial. Elaborate strategies are required to assure optimum overlap of the incident beam and the analyzer acceptance cone as the electron energies and the scattering angle are changed, and to determine the response function of the instrument for all the energies and angles required. Few research groups have constructed the magnetic angle changer and developed methods to achieve this goal $[3,4,5]$. But there are still inherent uncertainties which are sometimes apparatus specific. These procedures are essential for the measurements and are elaborated here in some detail for the instrument used in Fribourg. 

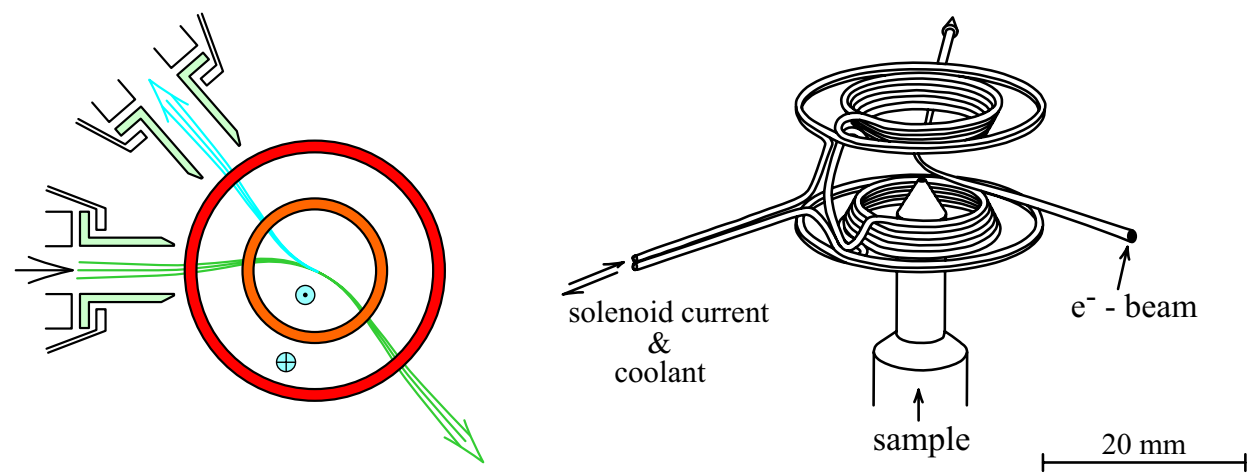

FIGURE 1. The Magnetic angle changer.

This paper reports on efforts to improve the methods to measure suitable sets of cross sections and illustrates the results on several molecules.

\section{EXPERIMENT}

The instrument, is a conventional electron spectrometer with two hemispherical electron monochromators and two hemispherical electron analyzers $[6,7,8]$. The instrument incorporates the magnetic angle changer (MAC) invented by Read and coworkers [1, 2]. The particular form of the MAC realized in Fribourg [9] is made of few windings of a thin $(0.63 \mathrm{~mm}$ diameter) copper tubing, cooled by water. This design minimizes the obstruction of the gas flow, the local pumping speed in the collision region is nearly unaffected by the presence of the MAC. This reduces beam attenuation by background gas, is important for absolute measurements which rely on a definite gas flow, and improves resolution by reducing thermal Doppler broadening encountered in scattering by the background gas.

It may appear that incorporating the MAC device solves all the problems and the measurement of cross sections over the entire angular range becomes straightforward. A naive use of the device can easily result in errors exceeding a factor of ten, however. The central issues are:

- Ascertaining an optimal overlap of the incident beam, the analyzer acceptance cone (the 'scattered beam'), and the gas beam, when the electron energies $E_{i}$ and $E_{r}$ and the scattering angle $\theta$ are changed.

- Determining the 'response function' of the instrument both as a function of the electron energies and the scattering angle and properly correcting the raw data.

These issues have been discussed in detail in reference [10].

Briefly, the response function for elastic signal is determined by measuring the $\mathrm{He}$ elastic signal as a function of energy and dividing it point by point by the theoretical cross section [11]. Care must be taken of the thermal Doppler broadening and shift of the elastic peak due to momentum transfer. A remaining problem is that the response function determined in this way applies to elastic scattering and applying it to inelastic 


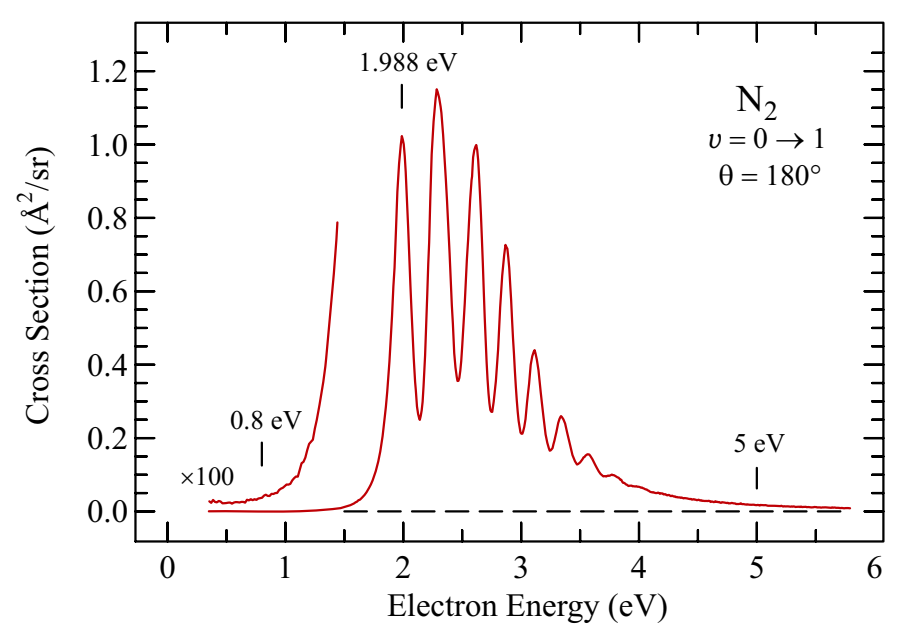

FIGURE 2. The rotationally summed $v=0 \rightarrow 1$ cross section at $180^{\circ}$

scattering represents an approximation which may not be entirely correct very close to threshold (within about $0.3 \mathrm{eV}$ ).

An important aspect of the measurement is 'tuning' of the instrument, that is determining a set of focusing and $x, y$-deflection voltages of the monochromator exit and analyzer entrance lens insuring optimal focusing and beam overlap over a range of electron energies and scattering angles. This is done by optimizing elastic and inelastic signals in helium, sometimes also in $\mathrm{CO}_{2}$ and $\mathrm{N}_{2}$, for a number of discrete electron energies and scattering angles (the 'pivotal points'). The computer then automatically interpolates these parameters between the pivotal points during acquisition.

Experience showed that the present instrument can not be optimized over the entire angular range at one time because of potential drifts. The entire angular range has been divided into three overlapping angular ranges by placing the analyzer at the angles of $\theta=45^{\circ}, 90^{\circ}$ and $135^{\circ}$, and sweeping the angle magnetically repetitively in a range of $\pm 45^{\circ}$ around the mechanical analyzer position. The partial angular measurements were then combined.

\section{RESULTS}

\section{Nitrogen}

A detailed account of the nitrogen cross sections measured with the spectrometer described above has been given in reference [10]. As an illustration of the capacity to measure at $180^{\circ}$ figure 2 shows the cross section for exciting the $v=1$ level, as a function of the energy of the incident electron. The current in the magnetic angle changer has been continuously adjusted by the computer to keep the scattering angle fixed at $180^{\circ}$ while changing the incident and scattered electron energies. The cross section is dominated by the well-known ${ }^{2} \Pi_{g}$ resonance centered around $2.3 \mathrm{eV}$.

Figures 3-5 show the cross sections recorded as a function of scattering angle at the 


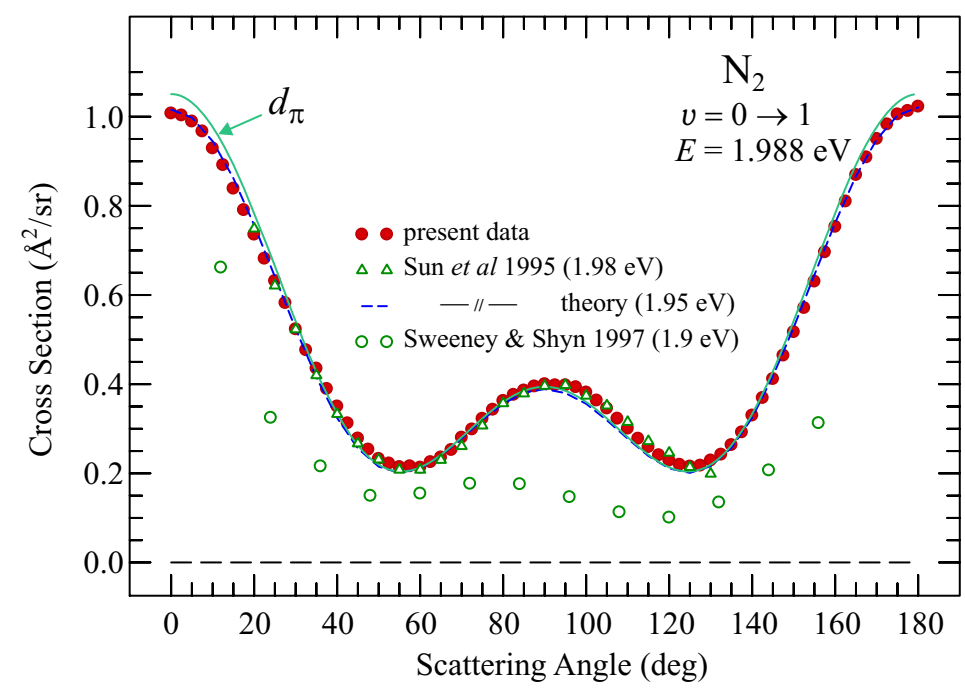

FIGURE 3. The rotationally summed $v=0 \rightarrow 1$ cross section at at the peak of the resonance region $(E=1.988 \mathrm{eV})$. The results of Sun et al. [12], Sweeney and Shyn [13], and the $d_{\pi}$ wave distribution (normalized to the experiment at $90^{\circ}$ ) are shown for comparison

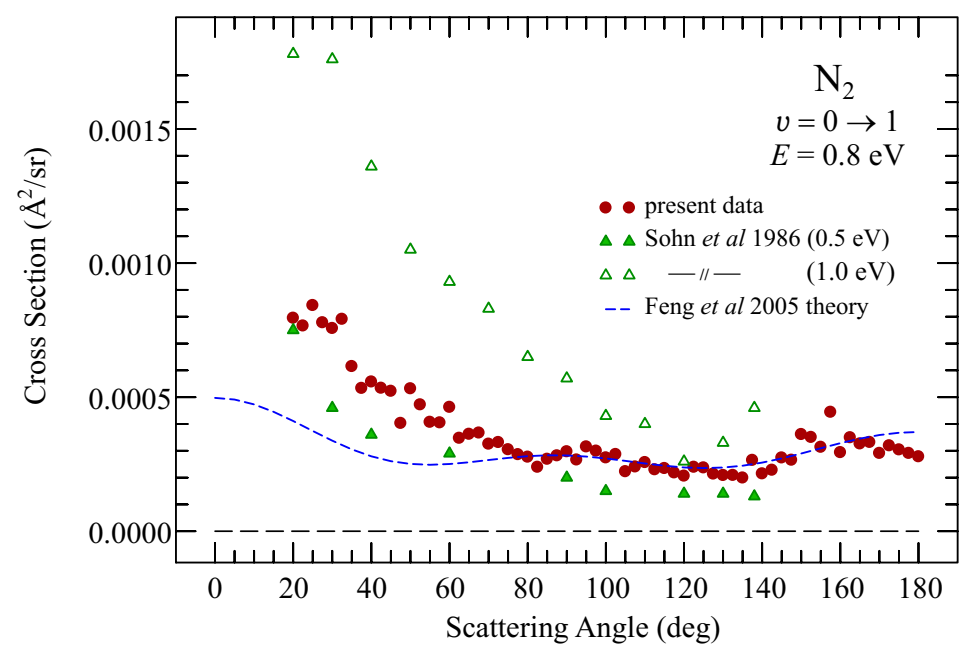

FIGURE 4. The rotationally summed $v=0 \rightarrow 1$ cross section below the resonance region (at $E=$ $0.8 \mathrm{eV}$ ). The experimental data at nearby energies of Sohn et al. [14], and the theoretical results of Feng et al. $[15,16]$ (DSG model) are shown for comparison.

three incident energies marked by bars in figure 2. One energy is taken exactly at the resonance, one below and one above.

The cross section taken at resonance agrees remarkably well with the calculation of Feng, Sun and Morrison given in reference [12]. The shape of the cross section agrees also very well with the analytical expression for a $d_{\pi}$ wave distribution, confirming the dominance of the ${ }^{2} \Pi_{g}$ resonance at this energy. In the common angular range there is an excellent agreement with the earlier experimental results of Sun et al. [12].

The cross section taken at $0.8 \mathrm{eV}$, below the resonance, is much smaller and much 


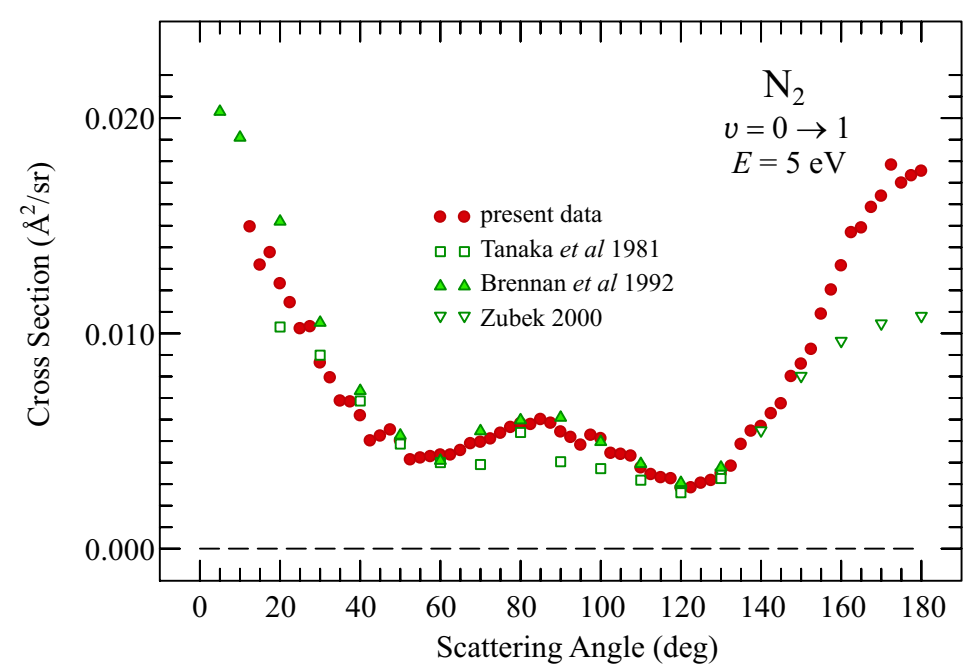

FIGURE 5. The rotationally summed $v=0 \rightarrow 1$ cross section above the resonance region (at $E=5 \mathrm{eV}$ ). The results of Tanaka et al. [17], Brennan et al. [18] and Zubek et al. [3] are shown for comparison.

more difficult to measure. In fact, the measurement failed below $20^{\circ}$ because background due to stray electrons increases in the forward direction. No older experimental data exists at $0.8 \mathrm{eV}$, but the present results can be compared - favorably - with the data of Sohn et al. [14] taken below and above this energy. The agreement with the calculation of Feng et al. $[15,16]$ is very good above about $60^{\circ}$.

The cross section at $5 \mathrm{eV}$ is larger than at $0.8 \mathrm{eV}$ and could be measured down to $10^{\circ}$. The shape still resembles the $d_{\pi}$ wave, indicating that the ${ }^{2} \Pi_{g}$ resonance is important even at $5 \mathrm{eV}$. There is a very good agreement with older experiments in the angular range $10-150^{\circ}$. The earlier data of Zubek et al. [3] is lower than the present data above $150^{\circ}$, but this difference has been resolved in the mean time [19].

The data in figures 3-5 has been used to derive the integral and momentum transfer cross sections which are generally in good agreement [10] with values obtained from electron transport measurements. Very good agreement of the integral cross section at $0.8 \mathrm{eV}$ was also found with the recent calculation of Telega and Gianturco [20].

\section{Methane and neopentane}

This section presents preliminary data on methane, $\mathrm{CH}_{4}$, and neopentane, $\mathrm{C}\left(\mathrm{CH}_{3}\right)_{4}$. The present elastic cross section measured at $90^{\circ}$ is compared to older measurements in figure 6. The good agreement further validates the data obtained in Fribourg. The Ramsauer-Townsend minimum, already visible in the data of Sohn et al. [21], is prominent.

Figure 7 compares the elastic cross sections measured at several scattering angles including $180^{\circ}$, where the magnetic angle changer was used. The Ramsauer-Townsend minimum is seen to move to lower energies and to become deeper with increasing scattering angle. A secondary minimum at higher energy starts to appear around $135^{\circ}$ 


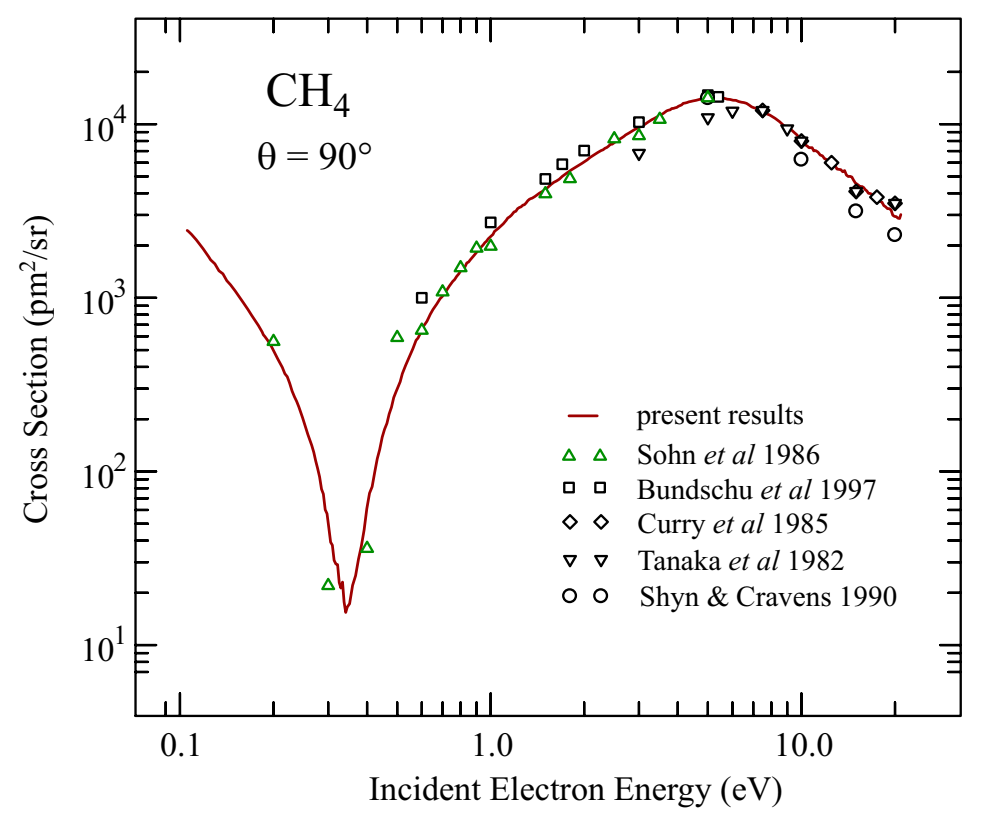

FIGURE 6. The $90^{\circ}$ elastic cross section in methane. The results of Sohn et al. [21], Bundschu et al. [22], Curry et al. [23], Tanaka et al. [24] and Shyn and Cravens [25] are shown for comparison.

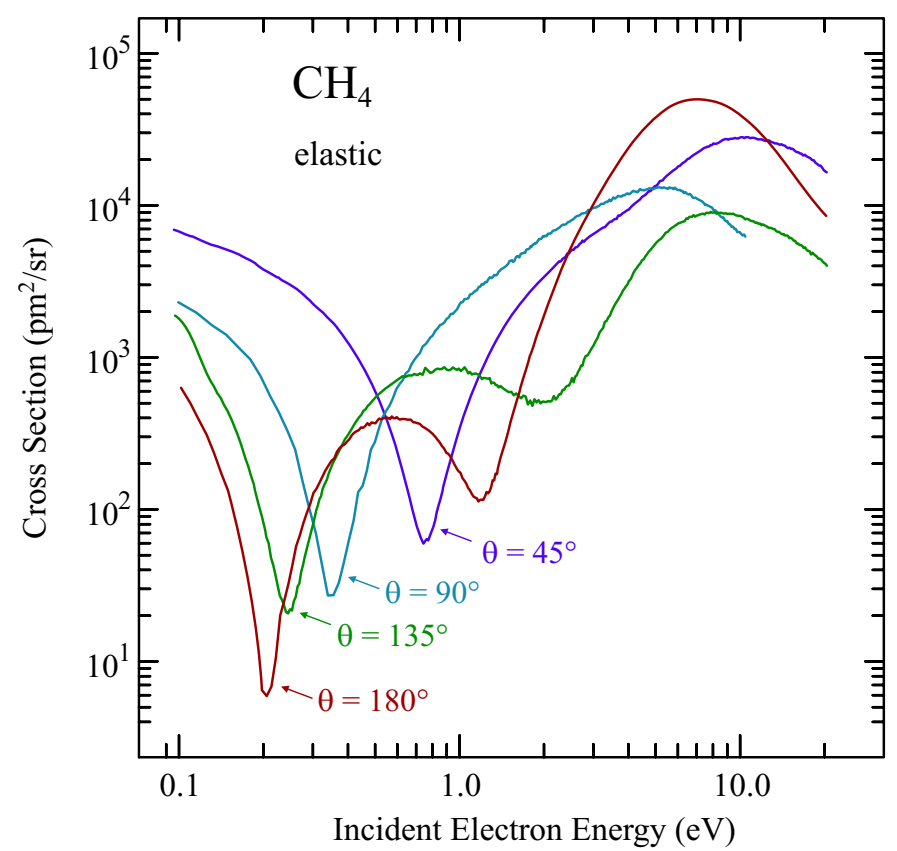

FIGURE 7. The elastic cross sections in methane at four representative angles.

and also moves to lower energies and becomes deeper with increasing scattering angle.

The presence of pronounced minima in the cross section is related to the high symmetry of methane and it therefore appeared interesting to study how do these minima behave when the size of the molecule is increased and the symmetry is kept high. This is 


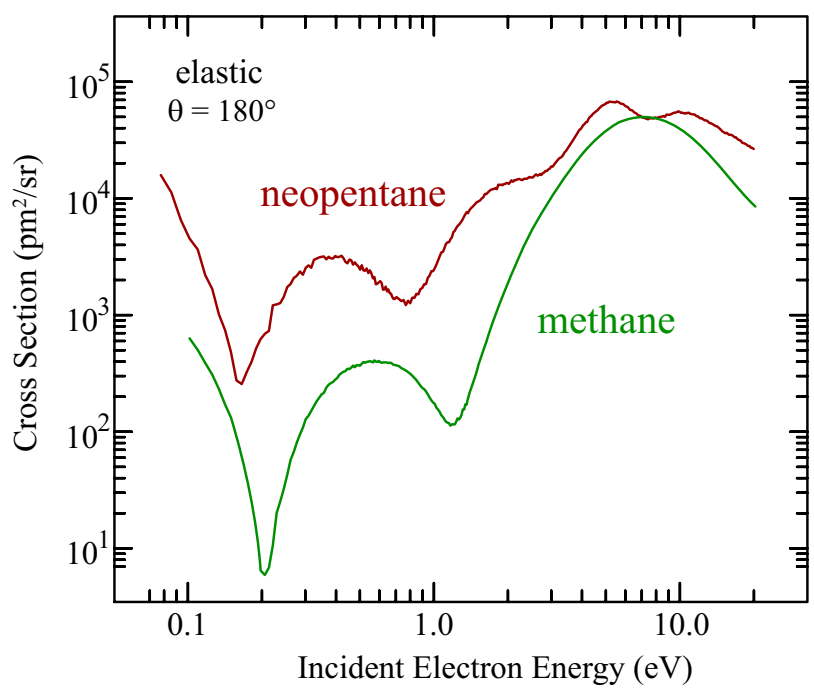

FIGURE 8. Comparison of the elastic cross sections in methane and neopentane at $180^{\circ}$.
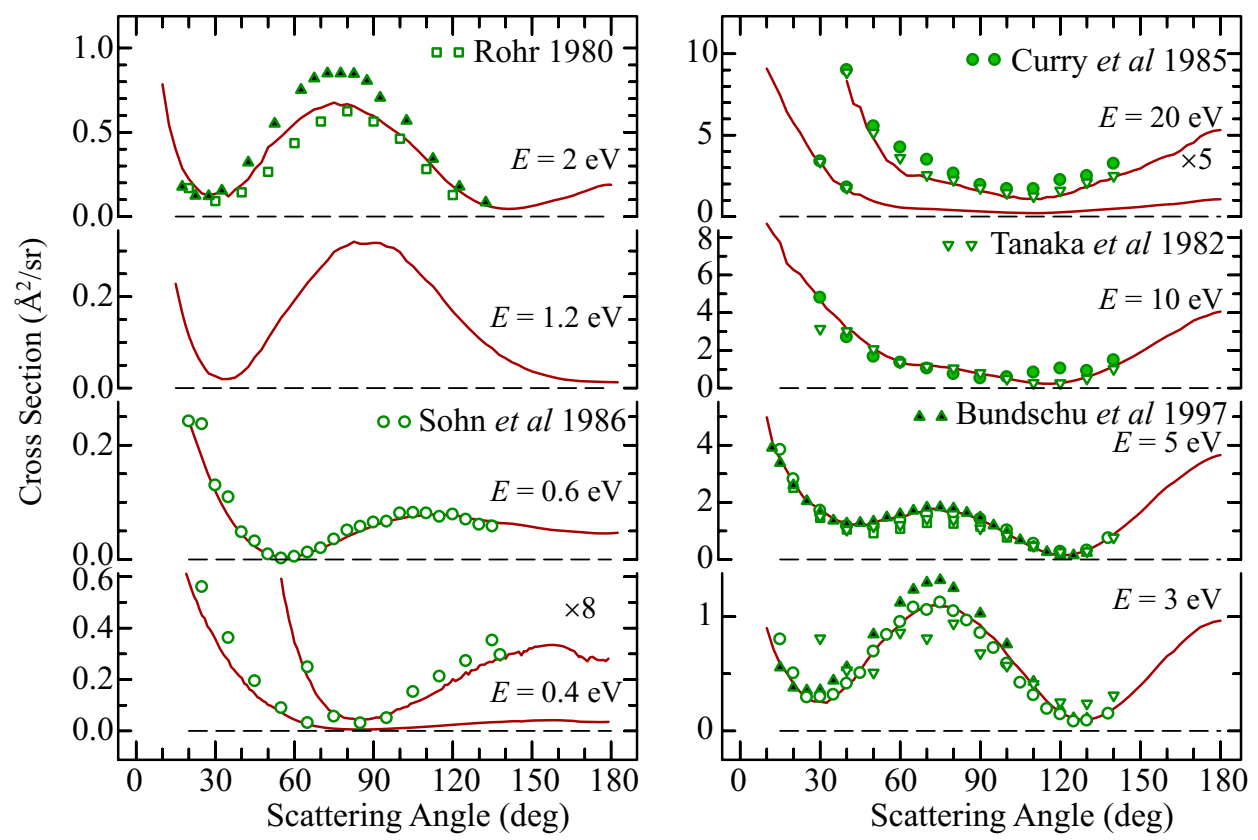

FIGURE 9. Elastic cross sections in methane. Present data is shown by continuous lines. The results of Sohn et al. [21], Rohr [26], Bundschu et al. [22], Tanaka et al. [24] and Curry et al. [23] are shown for comparison.

the case for neopentane which results when every hydrogen in methane is replaced by a methyl group. The elastic cross sections recorded at $180^{\circ}$ are compared in figure 8 . The neopentane cross section is generally larger as would be expected because of its larger size. The two minima observed in methane persist in neopentane, but move to slightly lower energies. Neopentane exhibits two more minima at even higher energies.

Figure 9 shows the elastic cross sections in methane. The agreement with previous 

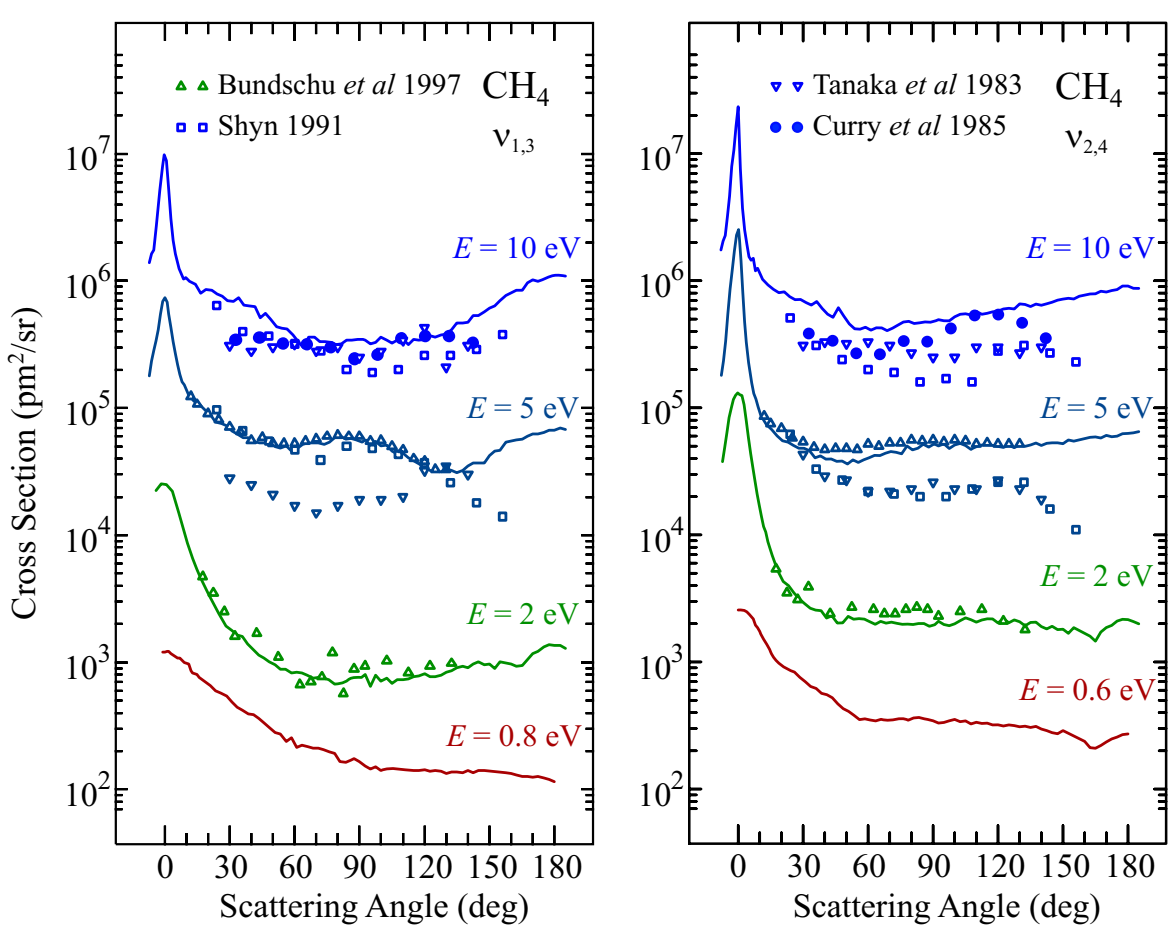

FIGURE 10. Cross sections for vibrational excitation in methane. Present data is shown by continuous lines. The vertical scales apply to the lowest spectra and the remaining spectra are offset upward for clarity, by a factor of ten for every higher energy. The results of Bundschu et al. [22], Shyn [27], Tanaka et al. [28] and Curry et al. [23] are shown for comparison.

data is generally satisfactory, but the present data extends the angular range. Noteworthy is that Sohn et al. [21] were able to measure accurately at remarkably low energies already 20 years ago.

Figure 10 shows the cross sections for vibrational excitation in methane. The vibrational peaks for the vibrations $v_{1}$ and $v_{3}$, and for $v_{2}$ and $v_{4}$ overlapped because of strong rotational excitation, particularly at higher energies, and the sums of the cross sections were therefore measured. The new data generally, but not always agrees with older measurements in the intermediate angular range. A very intense peak, sometimes a cross section increase of more than a factor of 10 , is observed around $0^{\circ}$ at higher energies.

\section{Electronic excitation of rare gases}

The methods and calibration procedures described above have been applied to measure cross sections for electronic excitation of neon, argon and xenon in the near threshold region $[29,30]$. This subject has been treated in detail by $\mathrm{K}$. Bartschat in this volume and will be mentioned here only briefly from the experimental point of view. The experimental data has been very useful to validate the recent significant progress in theory, made by means of a $B$-spline $R$-matrix method [31, 32, 33].

A representative spectrum is shown in figure 11, based on the data of reference [29]. 


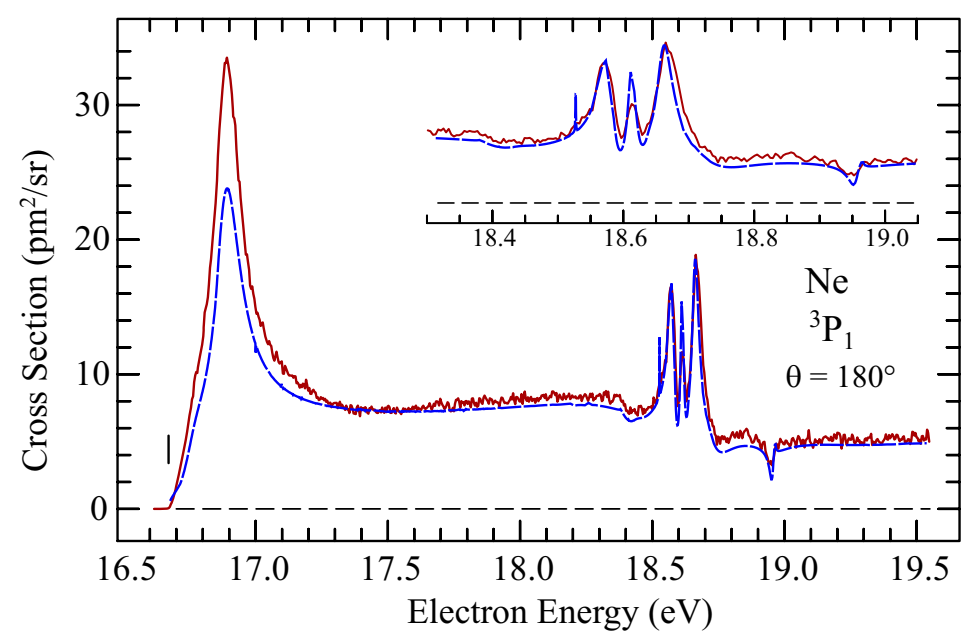

FIGURE 11. Experimental (line with statistical noise) and theoretical (smooth dashed line) cross section for exciting the ${ }^{3} \mathrm{P}_{1}$ state of neon. (based on reference [29])

The theoretical and experimental data agree remarkably well above about $17.3 \mathrm{eV}$, but the experimental cross section is slightly higher within the first about $0.4 \mathrm{eV}$ above threshold. This difference could be due to an overestimation of the instrumental response in this low energy range. This error could be consequence of using the elastic cross section (in helium) to derive the response function, which is then used to correct deeply inelastic data. To by-pass this problem, it would be useful to have very reliable theoretical inelastic cross section for helium which could be used as a secondary standard in determining the instrumental response function for inelastic scattering near threshold.

\section{CONCLUSIONS}

The measurements of absolute cross sections for electron scattering are now consistent between the leading groups in this research field. The angular range of the measurements has been extended by the magnetic angle changer. Reasonably large inelastic cross sections can now be measured over the entire angular range, elastic and very weak inelastic cross sections in the range of about $20^{\circ}-180^{\circ}$. Great care must be taken to take the instrumental response function and the instrumental drifts into account. The reliability of the measurement is indicated also by the good agreement of the angle-integrated (integral and momentum-transfer) cross sections with the results of electron-transfer experiments for nitrogen, even for the $v=0 \rightarrow 1$ cross section below the resonance, which is difficult to measure because of its small size. Two minima are observed at low energies in the elastic cross section of methane and neopentane (recorded in function of electron energy). The method has also been very successful in recording cross sections for electronic excitation of rare gas atoms in the near threshold region, where very good agreement with recent R-matrix theory has been achieved.

The drawback of the method is that measurements which include all scattering angles are very time consuming. 
Finally, it is recommended that all research groups in this field use the same standard for normalizing their data. For this purpose a set of state-of-the-art theoretical cross sections in helium, updating the 1979 data of Nesbet, would be desirable. It should extend from $0 \mathrm{eV}$ to at least $30 \mathrm{eV}$, and from $0^{\circ}-180^{\circ}$, on a dense mesh with respect to energy and angle, and be published numerically on the web. The elastic cross section is required primarily, but the reliability of the experimental data very close to threshold could be improved if inelastic cross sections, reliable enough to be used as a standard, could also be calculated.

\section{ACKNOWLEDGMENTS}

This research was supported by the Swiss National Science Foundation.

\section{REFERENCES}

1. F. H. Read, and J. M. Channing, Rev. Sci. Instrum. 67, 2373 (1996).

2. M. Zubek, N. Gulley, G. C. King, and F. H. Read, J. Phys. B 29, L239 (1996).

3. M. Zubek, B. Mielewska, and G. C. King, J. Phys. B 33, L527 (2000).

4. H. Cho, Y. S. Park, H. Tanaka, and S. J. Buckman, J. Phys. B 37, 625 (2004).

5. I. Linert, and M. Zubek, J. Phys. B 39, 4087 (2006).

6. M. Allan, Phys. Rev. Lett. 87, 033201 (2001).

7. M. Allan, J. Phys. B 25, 1559 (1992).

8. M. Allan, J. Phys. B 28, 5163 (1995).

9. M. Allan, J. Phys. B 33, L215 (2000).

10. M. Allan, J. Phys. B 38, 3655 (2005).

11. R. K. Nesbet, Phys. Rev. A 20, 58 (1979).

12. W. Sun, M. A. Morrison, W. A. Isaacs, A. D. T. Trail, W K, R. J. Gulley, M. J. Brennan, and S. J. Buckman, Phys. Rev. A 52, 1229 (1995).

13. C. J. Sweeney, and T. W. Shyn, Phys. Rev. A 56, 1348 (1997).

14. W. Sohn, K.-H. Kochen, K.-M. Scheuerlein, K. Jung, and H. Ehrhardt, J. Phys. B 19, 4017 (1986).

15. H. Feng, W. Sun, and M. A. Morrison, Phys. Rev. A 68, 062709 (2003).

16. H. Feng, W. Sun, and M. A. Morrison, private communication (2005).

17. H. Tanaka, T. Yamamoto, and T. Okada, J. Phys. B 14, 2081 (1981).

18. M. J. Brennan, D. T. Alle, P. Euripides, S. J. Buckman, and M. J. Brunger, J. Phys. B 25, 2669 (1992).

19. M. Zubek, private communication (2005).

20. S. Telega, and F. A. Gianturco, Eur. Phys. J. D 40, in press (2006).

21. W. Sohn, K. H. Kochem, K. M. Scheuerlein, K. Jung, and H. Ehrhardt, J. Phys. B 19, 3625 (1986).

22. C. T. Bundschu, J. C. Gibson, R. J. Gulley, M. J. Brunger, S. J. Buckman, N. Sanna, and F. A. Gianturco, J. Phys. B 30, 2239 (1997).

23. P. J. Curry, W. R. Newell, and A. C. H. Smith, J. Phys. B 18, 2303 (1985).

24. H. Tanaka, T. Okada, L. Boesten, T. Suzuki, T. Yamamoto, and M. Kubo, J. Phys. B 15, 3305-3319 (1982).

25. T. W. Shyn, and T. E. Cravens, J. Phys. B 23, 293 (1990).

26. K. Rohr, J. Phys. B 13, 4897 (1980).

27. T. W. Shyn, J. Phys. B 24, 5169 (1991).

28. H. Tanaka, M. Kubo, N. Onodera, and T. Suzuki, J. Phys. B 16, 2861 (1983).

29. M. Allan, K. Franz, H. Hotop, O. Zatsarinny, and K. Bartschat, J. Phys. B 39, L139 (2006).

30. M. Allan, O. Zatsarinny, and K. Bartschat, Phys. Rev. A 74, R030701 (2006).

31. O. Zatsarinny, and K. Bartschat, J. Phys. B 37, 2173 (2004).

32. O. Zatsarinny, and K. Bartschat, J. Phys. B 37, 4693 (2004).

33. O. Zatsarinny, and K. Bartschat, Phys. Rev. A 71, 022716 (2005). 